\title{
Calculation of radial inhomogeneity cylindrical shell when exposed to high temperatures by numerical-analytical method and fem
}

\author{
Vladimir Andreev ${ }^{1^{*}}$, and Lyudmila Polyakova ${ }^{1}$ \\ ${ }^{1}$ Moscow State University of Civil Engineering, 26 Yaroslavskoye Shosse, Moscow, 129337, Russia
}

\begin{abstract}
The purpose of the work is to compare two calculation methods using the example of solving the axisymmetric thermoelasticity problem. The calculation of a thick-walled cylindrical three-layer shell on the temperature effect was carried out by the numerical-analytical method and the finite element method implemented in the LIRA-CAD software package. In the calculation, a piecewise linear inhomogeneity of the shell due to its three-layer structure and continuous inhomogeneity caused by the influence of a stationary temperature field is taken into account. The numerical-analytical method of calculation involves the derivation of a resolving differential equation, which is solved by the sweep method, it is possible to take into account the nonlinear nature of the deformation of the material using the method of successive approximations. To solve this problem by the finite element method, a similar computational model of the shell was constructed in the LIRA-CAD software package. The solution of the problem of thermoelasticity for an infinite cylinder (under conditions of a plane deformed state) and for a cylinder of finite length with free ends is given. Comparison of the calculation results is carried out according to the obtained values of ring stresses.
\end{abstract}

\section{Introduction}

This article proposes a solution to the problem of thermoelasticity, taking into account changes in the properties of the material (concrete) depending on temperature and taking into account its physical nonlinearity. The mechanical characteristics of concrete at various temperatures were taken based on data from [1]. In work, a numerical-analytical method for solving this type of problem is described. This article proposes a comparative analysis of the calculation results by the numerical-analytical method and the finite element method implemented in the LIRA-CAD software package. The problem is solved both in the formulation of a plane deformed state (PDS) and for a finite cylinder with free ends.

* Corresponding author: asv@mgsu.ru 


\section{State of the problem}

The problem of calculating a three-layer cylindrical shell on the temperature effect is considered. Shell layer materials: the inner layer of heat-resistant concrete on alumina cement (concrete No. 1) $50 \mathrm{~mm}$ thick, the middle layer of heat-resistant concrete on Portland cement (concrete No. 2) - $100 \mathrm{~mm}$, the outer layer of steel - $40 \mathrm{~mm}$. A constant temperature of $500^{\circ} \mathrm{C}$ is maintained inside.

The dimensions of the shell layers and the temperature distribution inside the multilayer wall obtained by solving the heat equation are shown in Figure 1: $r_{1}=0.55 \mathrm{~m}, r_{2}=0.6 \mathrm{~m}$, $r_{3}=0.7 \mathrm{~m}, \quad r_{4}=0.74 \mathrm{~m} ; \quad T_{i}=500^{\circ} \mathrm{C}, \quad T_{1}=488,9^{\circ} \mathrm{C}, \quad T_{2}=380,4^{\circ} \mathrm{C}, \quad T_{3}=199,5^{\circ} \mathrm{C}$, $T_{4}=197,3^{\circ} \mathrm{C}, T_{o}=20^{\circ} \mathrm{C}$.
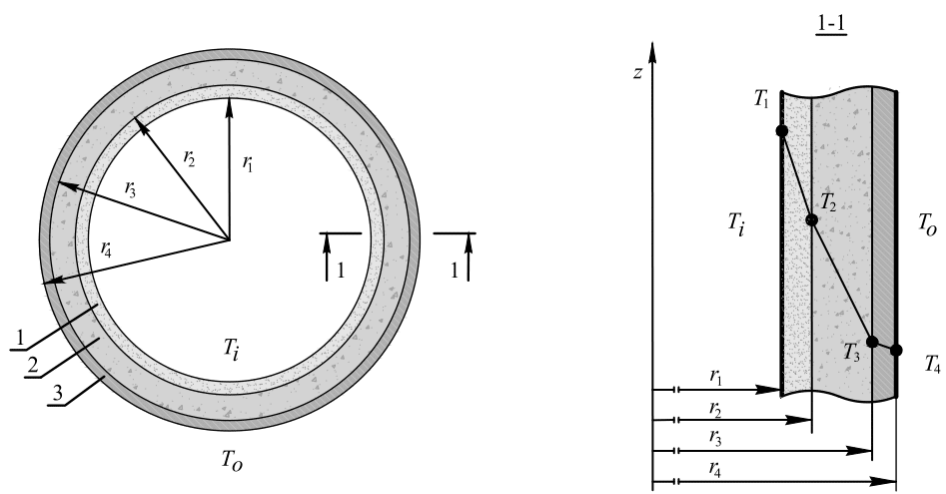

Fig. 1. Temperature distribution in a three-layer shell: 1 - concrete No. 1;2 - concrete No. 2; 3 - steel.

The change in the initial modulus of elasticity of concrete depending on temperature was taken according to the data from [1].

When solving by the numerical-analytical method to describe the nonlinear nature of the deformation of heat-resistant concrete, we used the experimental diagrams of the deformation of heat-resistant concrete, given in [2].

The solution uses a diagram $\sigma_{i}-\varepsilon_{i}$ that is described by a dependence with three constants, proposed in [3]:

$$
\sigma_{i}=E \varepsilon_{i}-A \varepsilon_{i}^{\alpha}
$$

The heterogeneity of concrete resulting from exposure to elevated temperatures is taken into account by replacing the constants $E, A$, and $\alpha$ with the functions $E(T), A(T)$, and $\alpha(T)$. In [4], the functions $E(T), A(T)$, and $\alpha(T)$ are given, which make it possible to approximate experimental diagrams of concrete deformation. To describe the deformation diagrams of heat-resistant concrete on Portland cement, the following functions were used:

$$
\begin{aligned}
& E(T)=E_{01}+k_{E 1}\left(\Delta T / T_{0}\right)^{0.5}+m_{E 1}\left(\Delta T / T_{0}\right)^{2.5} ; \\
& A(T)=A_{01}+k_{A 1}\left(\Delta T / T_{0}\right)^{0.8}+m_{A 1}\left(\Delta T / T_{0}\right)^{1.5} ; \\
& \alpha(T)=\alpha_{01}+k_{\alpha 1}\left(\Delta T / T_{0}\right)^{1.2}+m_{\alpha 1}\left(\Delta T / T_{0}\right)^{1.5} .
\end{aligned}
$$


Such formulas were successfully used earlier in [5,6] to describe nonlinear diagrams of concrete at high temperatures. In the presence of experimental curves, the choice of the coefficients in formulas (2) is not very difficult. To describe the diagrams deformation of heat-resistant concrete on aluminous cement authors in this paper suggest using the following functions:

$$
\begin{aligned}
& E(T)=E_{02} \cdot \exp \left[k_{E 2}\left(\Delta T / T_{0}\right)^{0.9}+m_{E 2}\left(\Delta T / T_{0}\right)^{0.2}\right] \\
& A(T)=A_{02} \cdot \exp \left[k_{A 2}\left(\Delta T / T_{0}\right)^{0.7}+m_{A 2}\left(\Delta T / T_{0}\right)^{0.3}\right] \\
& \alpha(T)=\alpha_{02} \cdot \exp \left[k_{\alpha 2}\left(\Delta T / T_{0}\right)^{0.9}+m_{\alpha 2}\left(\Delta T / T_{0}\right)^{0.15}\right] .
\end{aligned}
$$

In the formulas (2) and (3) $\Delta T=T-T_{0}$, where $T_{0}=20^{\circ} \mathrm{C}$ is the normal temperature of the concrete. The values of the coefficients are given in Table 1.

Table 1. The coefficients in formulas (1), (2)

\begin{tabular}{|c|c|c|c|}
\hline$E_{01}, \mathrm{MPa}$ & 47320 & $E_{02}, \mathrm{MPa}$ & 54720 \\
\hline$k_{E 1}$ & -7142 & $k_{E 2}$ & 0.14786 \\
\hline$m_{E 1}$ & 3.5 & $m_{E 2}$ & -0.655 \\
\hline$A_{01}, \mathrm{MPa}$ & 1700000 & $A_{02}, \mathrm{MPa}$ & 936300 \\
\hline$k_{A 1}$ & -169782 & $k_{A 2}$ & $3.05 \cdot 10^{-3}$ \\
\hline$m_{A 1}$ & 6285.14 & $m_{A 2}$ & -2.15856 \\
\hline$\alpha_{01}$ & 1.62 & $\alpha_{02}$ & 1.528 \\
\hline$k_{\alpha 1}$ & $2.0805 \cdot 10^{-2}$ & $k_{\alpha 2}$ & $-1.8306 \cdot 10^{-3}$ \\
\hline$m_{\alpha 1}$ & $-9.235 \cdot 10^{-3}$ & $m_{\alpha 2}$ & $-6.515 \cdot 10^{-2}$ \\
\hline
\end{tabular}

Also in the work [4] shows the form of these diagrams.

The values of the coefficient of linear temperature strain for concrete depending on the temperature were used according to the tables from, the task takes values $\alpha_{b}$ that correspond to the mode of prolonged heating under repeated exposure to temperature.

\section{Calculation method}

In [2], a numerical-analytical method for solving a plane axisymmetric problem for thickwalled shells of a physically nonlinear radially inhomogeneous material is described for arbitrary dependences of the mechanical characteristics on the radius. The method consists in solving a differential equation with respect to $\sigma_{r}$ the sweep method with a certain step; the method of successive approximations is used to take into account physical nonlinearity. When solving the differential equation, a constant step of $h=0.005 \mathrm{~m}$ was 
adopted, that is, a three-layer shell with a total thickness of $0.19 \mathrm{~m}$ is divided when solving into 38 ring layers, each of which corresponds to a set of parameters E, $v$ and $\alpha$; when physical nonlinearity is taken into account, a separate diagram $\sigma_{i}-\varepsilon_{i}$ is set for each layer.

The solution under the condition of flat deformed state assumes that the cylinder is very long and stresses arising at a sufficient distance from the ends are considered. A method for solving a similar problem with local perturbations near the ends of the cylinder is described in detail in [7].

To solve this problem by the finite element method, the LIRA-CAD software package was used. A model of the cylinder was created, divided by radius into 38 finite elements. 38 types of stiffness and temperature load were set for each annular layer.

When solving the plane problem, displacements along the $\mathrm{z}$ axis along the ends of the cylinder were limited. When solving the problem for the final cylinder, one end was released from fixing, while the total length of the cylinder was $1.45 \mathrm{~m}$, since a numericalanalytical calculation showed that stresses arising near the free ends of the cylinder quickly decrease with increasing distance from the end and at a distance of $1.5 \mathrm{~m}$ their influence is insignificant.

\section{Results}

Figure 2 shows a finite element model of a cylindrical shell with a free end deformed under the influence of temperature.

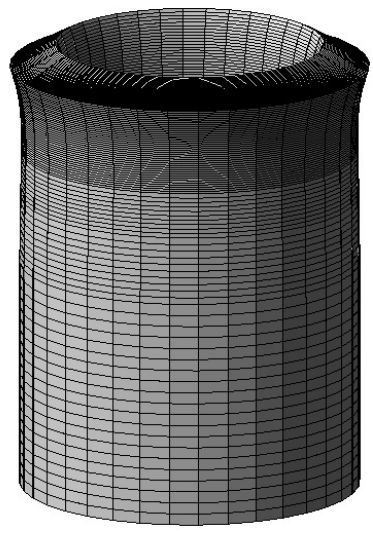

Fig. 2. The deformed cylindrical shell obtained in the LIRA-CAD program.

Figure 3 shows the stresses $\sigma_{\theta}$ distributed over the shell thickness at $z \geq 1.5 \mathrm{~m}: 1$ linear calculation by numerical-analytical method; 2 - linear calculation by the FE method; 3 - non-linear calculation by numerical-analytical method. Figure 4 shows the stresses $\sigma_{\theta}$ near the free end of the cylinder in the most stressed annular concrete layers at $r=0.55 \mathrm{~m}$ and $r=0.7 \mathrm{~m}: 1$ - linear calculation by numerical-analytical method, $r=0.7 \mathrm{~m}$; 2 - linear calculation by the FE method in the LIRA-SAPR program, $r=0.7 \mathrm{~m} ; 3$ - nonlinear calculation by numerical-analytical method, $r=0.7 \mathrm{~m} ; 4$ - linear calculation by numerical-analytical method, $r=0.55 \mathrm{~m} ; 5$ - linear calculation by the FE method in the LIRA-SAPR program, $r=0.55 \mathrm{~m} ; 6$ - non-linear calculation by numerical-analytical method, $r=0.55 \mathrm{~m}$. 


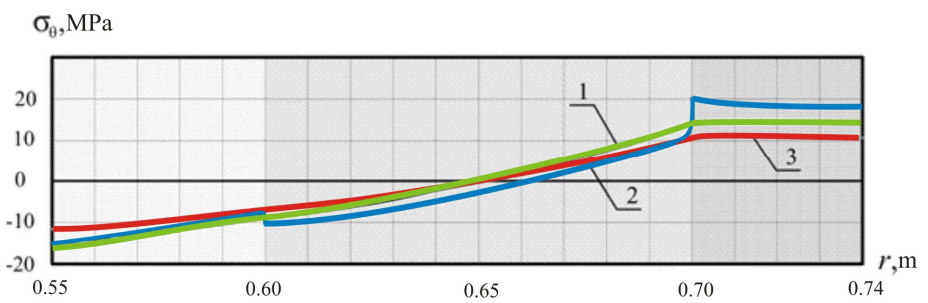

Fig. 3. Distribution of stress $\sigma_{\theta}$ along the thickness of the shell

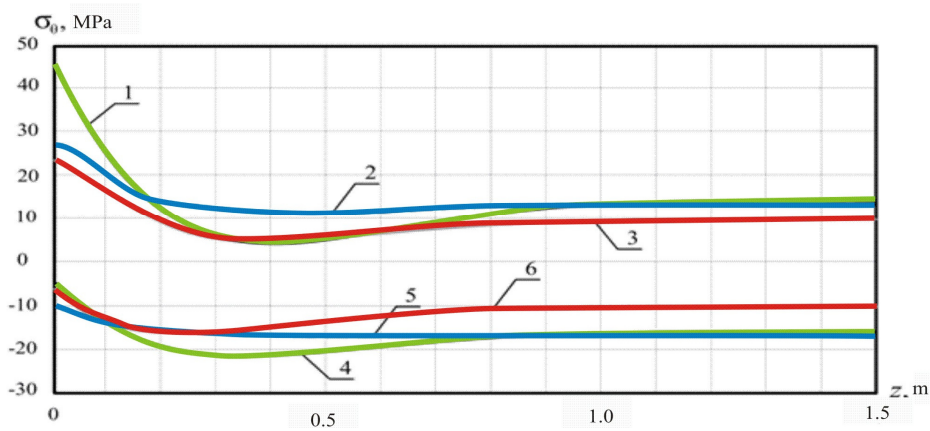

Fig. 4. Stresses $\sigma_{\theta}$ near the free end of the cylinder at $r=0.55 \mathrm{~m}$ (No 1-3)and $r=0.7 \mathrm{~m}$ (No 4 -6).

\section{Conclusions}

The results of solving the planar problem (Fig. 3) by the FE method in the LIRA-CAD program show sharp changes in stresses at the places of material change caused by the redistribution of stresses in favor of materials with a large modulus of elasticity, which are not present in the numerical analytical calculation, and should not be in the planar problem in the absence of deformations in $\mathrm{z}$.

The results of solving the problem of thermoelasticity of a cylinder of finite length show a significant discrepancy in the maximum stresses near the free end of the cylinder.

\section{References}

1. A.V. Ushakov, Basic laws of deformation of ordinary and heat-resistant concrete during heating (2006)

2. V.I. Andreev, L.S. Polyakova, Inte. J. Com. Ci. Struc. Eng. 12, 4 (2016)

3. Potekhin, V. Mischenko, A. Mottaeva, A. Zheltenkov, E3S Web of Conferences, 33, 03020 (2018) doi: 10.1051/e3sconf/20183303020

4. L.S. Polyakova, V.I. Andreev, IOP Conf. Series: Mate. Sci. Eng., 456, 012124 (2018)

5. V.I. Andreev, L.S. Polyakova Thermal stress of a Thick-walled Inhomogeneous Cylinder of Nonlinear-elastic Material, Advanced Materials and Structural Engineering (2016)

6. V. I. Andreev, L. S. Polyakova A. S. Avershyev, Proce. Engine. 153 (2016)

7. V.I. Andreev, L.S. Polyakova, E3S Web of conferences, 9102018 (2019) 